\title{
EFFICIENT HIGH QUALITY VIDEO ASSESSMENT USING SALIENT FEATURES
}

\author{
Bhanu Rekha $\mathrm{K}^{\mathbf{1}}$ and Ravi Kumar A. $\mathbf{V}^{\mathbf{2}}$ \\ ${ }^{I}$ Department of Electronics and Communication Engineering, Sir M. Visvesvaraya Institute of Technology, India \\ ${ }^{2}$ Department of Electronics and Communication Engineering, SJB Institute of Technology, India
}

\begin{abstract}
High Definition (HD) devices requires HD-videos for the effective uses of HD devices. However, it consists of some issues such as high storage capacity, limited battery power of high definition devices, long encoding time, and high computational complexity when it comes to the transmission, broadcasting and internet traffic. Many existing techniques consists these above-mentioned issues. Therefore, there is a need of an efficient technique, which reduces unnecessary amount of space, provides high compression rate and requires low bandwidth spectrum. Therefore, in the paper we have introduced an efficient video compression technique as modified HEVC coding based on saliency features to counter these existing drawbacks. We highlight first, on extracting features on the raw data and then compressed it largely. This technique makes our model powerful and provides effective performance in terms of compression. Our experiment results proves that our model provide better efficiency in terms of average PSNR, MSE and bitrate. Our experimental results outperforms all the existing techniques in terms of saliency map detection, AUC, NSS, KLD and JSD. The average AUC, NSS and KLD value by our proposed method are $0.846,1.702$ and 0.532 respectively which is very high compare to other existing technique.
\end{abstract}

Keywords:

HEVC, AUC, NSS, Encoding

\section{INTRODUCTION}

21st century has provided the practical world enormous innovative electronic inventions, which takes entire electronic research industry to a completely different level. Out of these outstanding achievements by the researchers, one quite popular and trending topic in today's market is High definition (HD) devices such as Action cameras, Ultra Edge high definition cameras and Ultra Edge TV etc. These high- definition (HD) devices becomes the mandatory practical requirement in the field of medical imaging, satellite imaging, face recognition systems, military, entertainment(for advertisements and movies), animation $3 \mathrm{D}$ view and images motion detection etc. For the vulnerable use of these high definition devices, there is need of high-resolution pictures and videos.

In recent years, the demand of high resolution videos drastically increased due to immense popularity of high definition devices such as Smart phones, iPad, MacBook Pro, LAPTOPS, HDTV (High-definition television), UHDTV (Ultra-highdefinition television) and 3-D TV systems. Therefore market is highly dominated by the requirement of high-resolution videos in the field of medical, photography, satellite imaging, HDTV, stereoscopic video processing, face recognition and video coding or encoding in recent few years.
However, it comes with some eye-catching issues such as high storage capacity, limited battery power of high definition devices, long encoding time, large bitrate degradation, and high computational complexity. This led to high bandwidth demand and need of large data storage in internet traffic [1]. Therefore, to minimize the memory and large bandwidth spectrum issue video encoding is a vital phenomenon. There are many algorithms presented in recent few years in the field of video encoding, as it is a hot topic from the prospect of industry and fast growing electronic market.

The encoding efficiency of high-resolution videos is quite low using earlier techniques. Therefore, to counter the problems the latest video encoding standard techniques H.264/AVC [2] and HEVC/H.265 [3] developed to reduce the size of high-resolution videos largely by producing large compression rates while maintaining the quality of videos. However, in real time it is very difficult to achieve high compression rates due to drastic increase in computational complexities. Another issue related to limited battery lifetime as to capture high-resolution videos, high definition battery-powered cameras required in the field of sports, travel, military, rescue, surveillance, entertainment, daily life and broadcasting high-definition videos. This issue can further led to the encoding bit-rate and distortion trade-off issue [4-12].

Therefore, to counter these issues in global interest many researchers has carried out some great amount of work in video encoding field. A very popular and fast growing technique in recent time for video encoding is H.264/AVC (Advanced Video Encoding) standards. This standard approach used to increase the compression rate for high-resolution videos. However, the computational complexity is very high for this standard, which causes its degradation in performance [2]. Another biggest disappointment with this technique is its slowness (takes more computational time) [13]. Map Reduce based distributed algorithm [13] used to speed-up the encoding process. However, it requires large amount of bandwidth to encode high definition videos. A one more technique, Adaptive Scheduling Framework for Real-Time Video Encoding presented in [14] to reduce the computational complexity. However, it requires parallelization at inter-loop level, which is very hard to achieve.

There is a huge demand of High Definition videos in the market due to availability of HD devices. However, there are many issues occur while transmitting, storing and live streaming of HD videos. Therefore, there is a need of efficient video encoding without degradation in video quality. However, it is very complex process to achieve efficient video encoding as it consists of many issues such as limited battery power of high definition devices, long encoding time, large bitrate degradation, limited bandwidth and high computational complexity problems. Although, a healthy research has been done to counter these 
drawbacks and generates a high quality video encoding. However, still it lacks an effective application to remove these drawbacks. Therefore, this motivates us to present an efficient technique, which can handle most of these issues effectively. Therefore, we have proposed a video compression technique modified HEVC coding based on a saliency features.

In many popular existing techniques, they first compress the video dataset and then extract their features. Therefore, the compression rate become very low as the data is already compressed which also reduces the performance of encoding process. Therefore, to eliminate this drawback and make the encoding performance better we first extract HEVC features and then compress the raw data to very low size. This phenomenon provides better compression, required bit-rate and faster compare to other algorithms.

This paper is organize in following sections, which are as follows. In section 2, we describe about the video encoding issues and how they can eliminate by our proposed model. In section 3 , we described our proposed methodology. In section 4, experimental results, evaluation shown, and section 5 concludes our paper.

\section{VIDEO ENCODING ISSUES}

However, very few techniques emerges as a practical solutions to above mentioned, limited battery power of high definition devices, long encoding time, large bitrate degradation, limited bandwidth and high computational complexity problems. Still, there is a need of some good research, which can efficiently target these eye-catching issues as they directly affects the performance of the encoding process. A brief of related work in the field of video encoding presented in the following section.

A Novel Bitrate-Saving and Fast Coding technique for depth video in 3D HEVC presented in [15] to reduce synthesis error, which produced while processing of depth, and color map encoding. This technique can be very effective to get 3-D view of high definition (HD) videos. However, it degrades the bitrate largely, which is quite difficult to achieve back completely. In [13], Segmentation and Scheduling video encoding based on distributed Map Reduce architecture presented to speed up the encoding process by using advanced distributed technique. In this paper, video segmentation technique used to provide better efficiency and to change the encoding segment order an efficient scheduling scheme adopted. However, it generates high bitrate and reduces the efficiency of the content differentiation process, which can led to performance degrade in encoding process. In [16], a Video Encoding technique based on Content-Aware approach presented to provide a consistent quality of encoded video and required bitrate to satisfy bandwidth necessities. The main idea behind this is to reduce computational complexity by searching frame wise optimal quantization factors, which rely upon content-based features and single pass encoding method to analysis distortion prototype. However, it requires high computational speed, high storage capacity and modern machinery with multicore design to handle large computations. In [14], a Real-Time Video Encoding for Heterogeneous Systems adopted based on Adaptive Scheduling Framework to provide real-time encoding by using a combination of CPU+GPU cores parallel for heterogeneous devices. However, heterogeneous devices consists of unified optimization problem in video encoding and provides high computation complexity. In [17], Key point Encoding for Improved Feature Extraction from Compressed Video at Low Bitrates performed to detect key-points and descriptor calculation, which helps in detecting different variations, occurs in video encoding, to lower bitrates. In this paper, they have reduced bitrate to a sufficient amount for a single scene of a video. However, it can be very complex process for multiple scenes of a video and bitrate can be very high to encode multiple scenes of a video. In [18], HEVC Coding presented based on Fast Intra Prediction to reduce the computational complexity and bitrate while video encoding. in this paper, Screen content coding (SCC) used to provide low latency and fast transmission with HEVC technique. However, it consists of larger prediction units, which can cause very high complexity, and the motion estimation of a video can be an expensive process. In addition, it requires high storage capacity and real camera capture contents to produce efficient outcomes in terms of bitrate and computational complexity. In [19], HEVC Live Video Encoding presented to reduce high computational complexity produced in HEVC technique and maintain quality of video encoding after reducing computational complexities. However, transmission bandwidth and delay consideration should be minimum (super-fast) for live video encoding using HEVC, which is very hard to achieve. Therefore, it can concluded from the above literature in the encoding field that still a healthy amount of work required countering all the video encoding issues effectively.

In this section, many algorithms presented as a related work. However, each algorithm has its own issues. The basic issues are high storage capacity, limited battery power of high definition devices, long encoding time, large bitrate degradation, and high computational complexity. Therefore, to overcome the issues we have proposed here video compression technique modified HEVC coding based on a saliency features. In this model, first we extract features on the raw data and then compress the raw data largely. This technique can increase the compression rate, takes less time to execute, and requires low computational complexity. This factor makes our proposed model more efficient compare to other existing techniques.

\section{PROPOSED METHODOLOGY}

\subsection{VIDEO ENCODING BASED ON SALIENCY FEATURES}

In recent years, the demand of high-definition videos has taken drastic growth in real practical world due to the presence of highdefinition devices. However, high-definition videos takes large space and bandwidth spectrum. Therefore, there is a need of an efficient video encoding which can maintain quality of a video without any data loss. Therefore, we have implemented a video compression technique based on a saliency features. This technique can used in field of medical, photography, satellite imaging, HDTV, stereoscopic video processing, face recognition and video coding or encoding to estimate saliency and compress high-definition videos largely. Our video compression provides fast computation for large training database such as SFU dataset [18] and HEVC video_database [19]. There are multiple factors, which makes HEVC architecture efficient and helps in enhancing the performance of the system. 
- This architecture helps to achieve lower bitrate, which helps in enhancing the performance of the encoding process.

- It can effectively estimate video saliency by extracting features efficiently.

- It takes less time while compression of large datasets such as SFU dataset [18] and HEVC video_database [19].

\subsection{VIDEO COMPRESSION USING SALIENCY FEATURES}

There are very few techniques, which can effectively estimate saliency and compress a high definition video largely without any loss in the quality. Therefore, to detect saliency efficiently and to provide high quality video after compression, we have implemented a video compression technique as modified HEVC based on a saliency features.

\subsection{COMPRESSED DOMAIN FEATURES}

There are many factors, which can make video compression very effective such as prediction of motion and motion compensation, then transformation, quantization, estimated residuals and motion vectors entropy. In recent years, many techniques evolved which tried to compress videos effectively without any loss of quality. Therefore, these factors becomes more advance with the evolution of new techniques. The outcomes of these factors provides compression domain features. These compressed domain features can work efficiently with the H.264/AVC standard [21] and HEVC (High Efficiency Video Coding) [20]. Our methodology involves $16 \times 16$ blocks, INTER, SKIP or INTRA processing blocks for different sizes $(4 \times 4,8 \times 8)$ and approximation of Transform for HEVC coding standard.

\subsubsection{Motion Vector Entropy (MVE):}

In video compression field, Motion Vector (MV) plays a very important role related to variations in the scene. It is a twodimensional block vector $\left(D_{a}, D_{b}\right)$ which balances the effect of best-matching template of a reference frame. Motion detection helps to find best-matching template. The approximation of Optical Flow provides a Motion Vector field.

Many different type of Motion Vectors are generated while video compression. Many different MVs created, when a moving object passed to a definite section in the video scene. These MVs generated in the corresponding spatial-temporal adjacent region. Some MVs represent moving object itself and some represents the background environment, out of these generated MVs. The Motion Vector of object can differ from each other. The Motion Vector of the background can cover entire section of the scene and provides consistent MVs. It derived from motion of camera. This change in MVs in the spatial-temporal adjacent region can utilized to recognize the existence of moving objects.

The processing of blocks inside a frame described as follows. This process carried out only for INTER blocks. However, SKIP blocks contains a zero Motion vector. All the Motion Vector (MVs) inside a frame mapped into $4 \times 4$ blocks. For example, we consider a $8 \times 8$ block for which a MV assigned. This MV can be distributed to all four constitutes blocks of size $4 \times 4$.

The motion vector entropy can described by the Eq.(1) as follows,

$$
m v e(x)=-\log M^{-1} \sum_{j=K(\theta(x))} m_{j} M^{-1} \cdot \log m_{j} M^{-1}
$$

Here, $x$ represent a block and $\theta(x)$ represents a motion cube linked with $4 \times 4$ block, $m_{j}$ is the number of motion vectors present in the bin index $j$. The parameter $\log M^{-1}$ in Eq.(1) lies between 0 (min) and $1(\max )$.

$$
\log M^{-1}=\left\{\begin{array}{c}
1 \max ; m_{j}=m_{k}, \forall j, k \\
0 \quad \min ; m_{j}=0, \forall j \neq 1
\end{array}\right.
$$

Background of a video scene can designed by large blocks and smaller blocks used to represent the moving objects with the help of motion estimation [22].

$$
\begin{gathered}
S_{m}=F_{d} \odot F_{n} \\
F_{d}(m)= \begin{cases}\frac{z}{T} & z \leq T \\
1 & z>T\end{cases}
\end{gathered}
$$

Here, Eq.(4) represents the element wise multiplication of motion magnitude $F_{d}$ and global angle $F_{n}$. The matrix for motion magnitude derived from a constant duration of a scene. Here, motion vectors used to construct saliency map. For every motion vector, a temporal-spatial motion magnitude calculated.

$$
F_{n}(m)=\frac{-P(\varphi) \cdot \log P(\varphi)}{\log m}
$$

Here, Eq.(5), represents the normalization to filter the inline motion vectors (MVs). In Eq.(2), $z$ is the motion magnitude after filtering of block $m$. T Represents the threshold. When $F_{d}$ becomes 1 , then it shows that the corresponding blocks consists large motion magnitude.

Here, Eq.(3) represents the normalized entropy to calculate the coherence global angle for motion vector. Here, $P(\varphi)$ represents the normalized histograms of motion vectors and $m$ denotes the number of histograms presents. To keep normalized histogram range in between 0 and $1, \operatorname{logm}$ used in denominator. If the normalized histograms of motion vector is 0 then it shows that camera motion is very high and if it is 1 then it shows motion inconsistency.

\subsubsection{Smooth Residual Feature:}

The motion-estimation residual detects that the current block is not same as the best-matching block in the reference frame. This shows that the block translation process cannot estimate motion of current block. This is due to either occlusions or large motion. However, most often it occurs due to occlusions. Occlusion can occur when suddenly any moving object comes in between the scene or hide behind the object. Therefore, large residuals can detect the sections where occlusions can occur.

The size of residual depends on the normalization function $n_{0}$ which is a non-zero variable. Residual Normalization feature can expressed in Eq.(3) as,

$$
R F(N)=256^{-1}\|N\|
$$

Here, $N$ represent the residual transformation of $16 \times 16$ macro block. Then, the spatial smoothness of residual normalization feature map calculated using $3 \times 3$ filter and temporal smoothness obtained by utilizing average moving filter over total existing frames. Therefore, a SRF (Smooth Residual Feature) map generated using $4 \times 4$ block. 


\subsubsection{Proposed Saliency Estimation:}

Here, we explain that how to estimate saliency using our proposed method. The detection of saliency can done without reconstructing the full video. This can verified by identifying two visual correlates of video fixation features for compressed territory. These two visual correlates are Motion Vector Entropy (MVE) and Smooth Residual Feature (SMF). Here, Fig.1 demonstrates the block diagram to compute and estimate saliency. For every frame, Motion Vectors (MV) and SRF (Smooth Residual Feature) and BCM (Block Coding Maps) generated. Here, RaceHorseD video data taken to obtain saliency map from HEVC video_database [19], [31-32]. Both the features are necessary to obtain a saliency map of an input video. In Fig.1 all the processing stages described to find saliency map.

To detect saliency map, many feature extraction approaches examined. [23, 24]. There are two factors which decides the accuracy of feature extraction process such as feature independency and behavior of their mutual activities which can affect the saliency map generation. MVE AND SRF values does not corresponds to each other i. e. one can be high and other can be low or vise-versa. The combination of both the features MVE and SRF can be very crucial which can give a better saliency map. If both the features are having huge values then there is very high possibility of moving objects in that region as well as it can contain sudden objects. Therefore, our model consists of a combination of both MVE and SRN features. The Eq.(7) represent the saliency map estimation.

$$
S M=\eta(m v e+R F(N)+m v e \odot R F(N))
$$

Here, $\odot$ represents the pointwise multiplication and $\eta(\cdot)$ represents the norm of range $[0,1]$. This proposed method is a combination of MVE and SRF to generate and estimate saliency map.

The Fig. 1 represent the block diagram to find saliency map using the combination of MVE and SRF feature. All the processing can done on HEVC coding standard. Most of the popular existing techniques, first they extract HEVC (High Efficiency Video Coding) feature then they compress the compressed outcome of HEVC feature. Therefore, there is very less area remain to further, compress on already compressed data. It also takes more time to extract features and encode the huge size data. These led to presence of high computational complexity. Therefore, to eliminate these drawbacks here we have first compressed our input raw video, then we extract features using modified HEVC coding standard on the processed input data. These phenomenon's increase our encoding performances by saving computational time and reducing computational complexity. Due to these factors, the compression rate will become very high. Therefore, our proposed model becomes more efficient than the existing techniques which verified by our experimental results shown in following sections.

\subsection{ACCURACY EVALUATION}

There are many parameters, which used to calculate the accuracy of visual saliency maps. Some of the parameters are AUC (Area under Curve), LCC (Linear Correlation Coefficient), JSD (Jensen-Shannon divergence), KLD (Kullback -Leibler divergence) and NSS (Normalized scan path saliency) in terms of gaze point information [33, 34, and 35]. All these parameters has its own importance with respect to evolution of performance. A prototype, which provides, better scores for all these mentioned parameters considered as an accurate prototype.

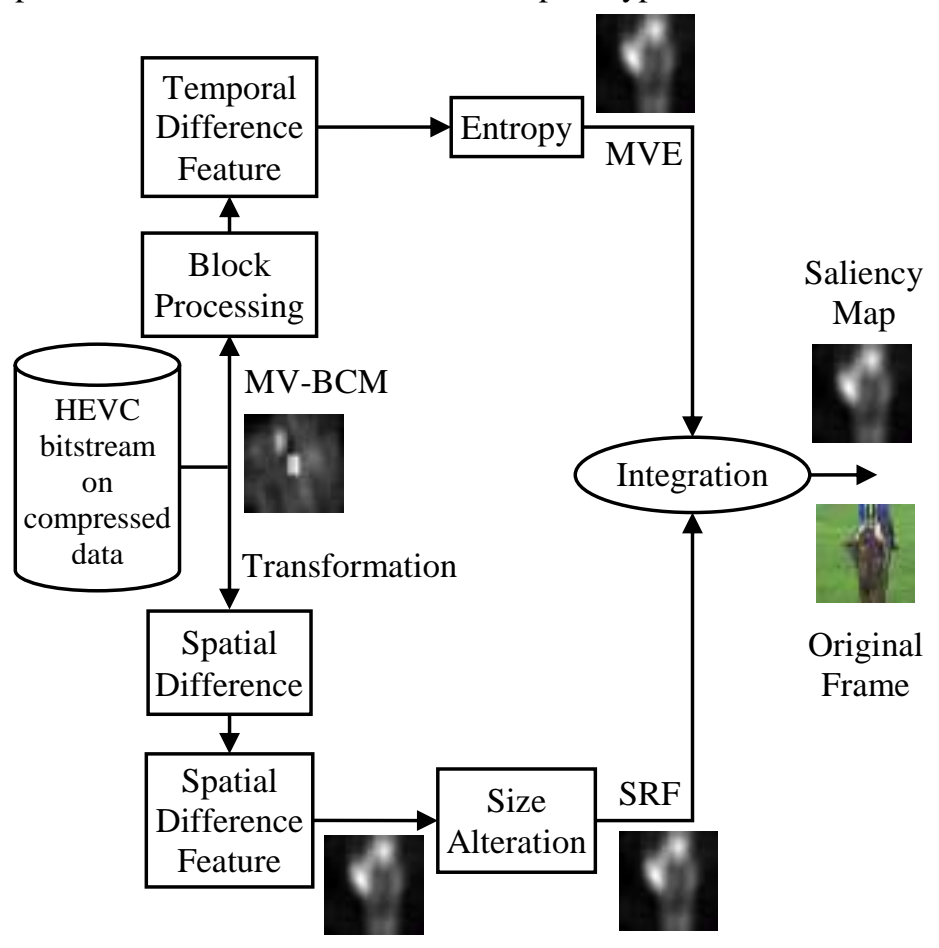

Fig.1. Block Diagram to Find Saliency Map using HEVC standard on compressed data

\subsubsection{Area Under Curve (AUC):}

AUC represents the properties of ROC (Receiver Operating Characteristic), more accurately, area under ROC curve, which can evaluated from the TPR (True Positive Rate) and FPR (False Positive Rate) at different threshold values. This parameter used to estimate performance and saliency map using gaze points. The AUC lies between 0 and 1. The lower value of AUC represents that the weaker saliency prediction and the higher value represents the better correspondence of saliency map.

\subsubsection{Kullback-Leibler Divergence (KLD):}

The parameter KLD used to find the divergence of two probability functions. It can defined as the relative entropy from one to another distribution. The higher values of KLD and JD can predict better saliency map or gaze points to evaluate accuracy.

$$
\begin{aligned}
& k l d(P \| Q)=\sum_{k} P(k) \cdot \log _{c} \frac{(P(k))}{(Q(k))} \\
& j d(P \| Q)=k l d(P \| R)+k l d(Q \| R)
\end{aligned}
$$

\subsubsection{SD (Jensen-Shannon Divergence):}

Jensen-Shannon divergence (JSD) is an upgraded version of $k l d$ (Kullback -Leibler divergence) to remove the drawbacks of $k l d$ AND $j d$. Its value ranges from 0 to 1 . The Eq.(10) represents the Jensen-Shannon divergence to evaluate performance using $Q$ and $P$ probability distributions.

$$
j d(P \| Q)=\frac{k l d(P \| R)+k l d(Q \| R)}{2}
$$




$$
R=\frac{P+Q}{2}
$$

\subsubsection{Normalized Scan Path Saliency (NSS):}

Normalized scan path saliency (NSS) used to find the average normalized saliency values at gaze positions. NSS consider parallelism to get standard deviation as 1 and mean as 0 . A positive value of normalized saliency at a definite gaze point shows the similarity of gaze points with the estimated salient fields while negative value of normalized saliency shows that the gaze points placed into a non-salient region. There is no relation between estimation and gaze points if value becomes zero.

\subsubsection{Linear Correlation Coefficient (LCC):}

Linear Correlation Coefficient used to evaluate the linear relationship between an estimated saliency and ground truth. The ground truth is defined as the convolution of gaze points and Gaussian functions with $\mathrm{SD}=1$. The linear correlation coefficient can evaluated as,

$$
\operatorname{corr}\left(G, S_{m}\right)=\frac{\operatorname{conv}\left(G, S_{m}\right)}{\sigma_{G} \sigma_{S_{m}}}
$$

Here, $\operatorname{conv}\left(G, S_{m}\right)$ represents covariance of $\sigma_{G}$ and $\sigma_{S_{m}}$. LCC ranges from -1 to 1 . In LCC, 0 shows no correction whereas \pm 1 shows strong bonding.

\section{RESULT AND ANALYSIS}

We compute our outcomes with the similar dataset as used in [18-19] to compare the performance and efficiency of our model to the existing techniques discussed in the related work. Our model trained on different large dataset like SFU dataset [18] and HEVC video_database [19], [31-32]. Here, we have shown results only for HEVC video_database [19]. Testing results shows that our model outperforms most of the existing techniques in terms of PSNR, feature extraction, compression rate and the estimation of saliency map. We have tested our model for different coding standards HEVC and H. 264/AVC. Our results shows accuracy, speed (bit rate) and Compression ratio enhancement largely. Our model needs less amount of execution time to provide effective video compression. Our model implemented on 64-bit windows 10 OS with 16GB RAM which consists on INTEL (R) core (TM) i5-4460 processor. It consists of 3.2GHz CPU. We have compared our model with Itti [24], Surprise [25], Judd [26], PQFT [27], Rudoy [28], Fang [29] and OBDL [30] existing techniques.

\subsection{IMPLEMENTATION DETAILS}

We have implemented our extensive experiments on large video SFU dataset [18] and HEVC video_database [19], [31-32]. In modern era, the availability of $4 \mathrm{~K}$ monitors is highly increased. Therefore, there is a huge demand of low-resolution videos to high-resolution videos in market. However, there is a huge problem of limited storage capacity and bandwidth spectrum. Therefore, there is need of efficient compression without loss of any kind of data and maintaining the high quality of the video. In this paper, we have first compressed the raw videos to large extent and then we extract HEVC features to get high quality of a video after effective compression. This technique saves large computational time and reduces high computational complexities.
To compute the performance of proposed modified HEVC model, here we have used 33 raw videos from the testing dataset [31-32] and 15 raw videos from the testing dataset [18]. All the experiments undertaken on the MATLAB 2016b framework.

\subsection{COMPARATIVE STUDY}

In this paper, we have compared our experimental results with many existing techniques such as Itti [24], Surprise [25], Judd [26], PQFT [27], Rudoy [28], Fang [29] and OBDL [30] existing techniques. In this paper, all the raw videos sampled on YUV 4:2:0 sampling. All the videos are compressed to high quality (more than $35 \mathrm{~dB}$ ). The resolution of input raw videos vary from $416 \times 240(240 p)$ and $1920 \times 1080$ (1080p). Performance metrics comparison is given in Table.1.

Table.1. Comparison of Performance Matrices with Existing Techniques for all four Videos in HEVC Video_Database Dataset with Ps

\begin{tabular}{|c|c|c|c|c|}
\hline Parameter & AUC & NSS & LCC & KL \\
\hline Itti [24] & 0.668 & 0.445 & 0.119 & 0.104 \\
\hline Surprise [25] & 0.752 & 1.078 & 0.272 & 0.183 \\
\hline Judd [26] & 0.816 & 1.427 & 0.187 & 0.285 \\
\hline PQFT [27] & 0.75 & 1.3 & 0.311 & 0.239 \\
\hline Rudoy [28] & 0.785 & 1.401 & 0.386 & 0.269 \\
\hline Fang [29] & 0.797 & 1.306 & 0.37 & 0.266 \\
\hline OBDL [30] & 0.785 & 1.511 & 0.352 & 0.236 \\
\hline HEVC [19] & 0.823 & 1.658 & 0.438 & 0.300 \\
\hline PS & 0.846 & 1.702 & 0.388 & 0.532 \\
\hline
\end{tabular}

Table.2. Comparison of Jsd for Different Videos of a Video_Database Dataset

\begin{tabular}{|c|c|c|c|c|}
\hline Parameter & Racehorsed & Male & Bqterrace & Slideshow \\
\hline JSD & 0.5033 & 0.480 & 0.4109 & 0.615 \\
\hline
\end{tabular}

The Table. 2 represents the performance comparison of our model with other existing techniques in terms of performance matrices such as AUC, NSS, LCC and KL. The average AUC (Area under Curve) value is very high using our proposed method compare to other existing techniques considering HEVC video_database dataset. The average AUC value by our proposed method is 0.846. Similarly, the average NSS (Normalized scan path saliency) and KLD (Kullback -Leibler divergence) value is very high using our proposed method compare to other existing techniques considering HEVC video_database dataset.

The average NSS and KLD value by our proposed method are 1.702 and 0.532 respectively. However, Linear Correlation Coefficient (Kullback -Leibler divergence) is an exceptional case in our proposed method as its value is little less. Overall our model performs very efficiently and outperforms all the existing techniques in terms of saliency map generation and compression. Similarly, we have also computed the advance JSD (JensenShannon divergence) parameter to detect saliency map more precisely. Here, in Table.2 JSD parameter computed for four videos. The Slideshow video gives highest JSD as 0.615 . 
Table.3. Saliency map comparison of all existing techniques with our proposed method for all four videos

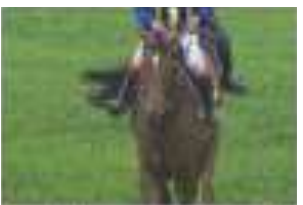

(a) Input

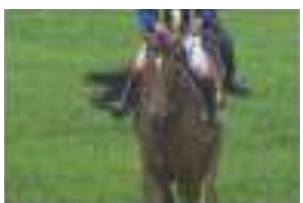

(a) Input

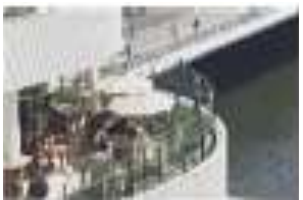

(a) Input

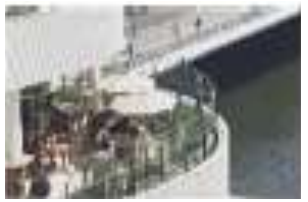

(a) Input

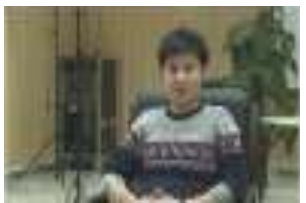

(a) Input

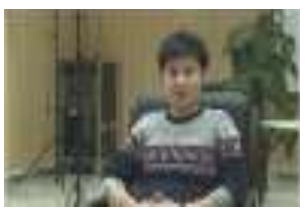

(a) Input

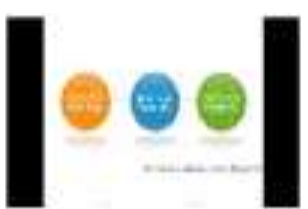

(a) Input

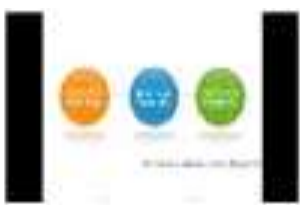

(a) Input

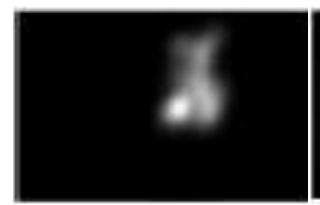

(b) Human

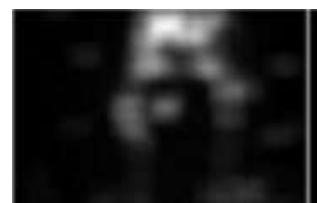

(g) PQFT

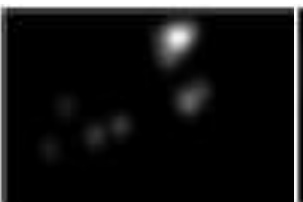

(b) Human

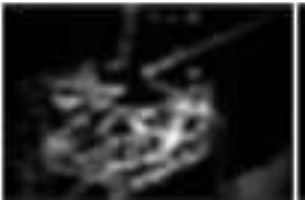

(g) PQFT

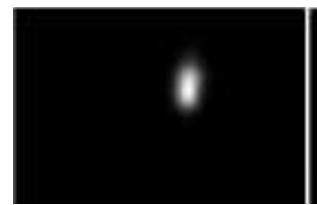

(b) Human

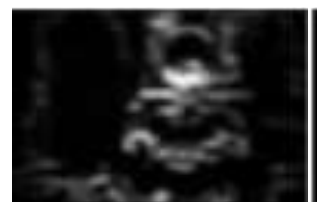

(g) PQFT

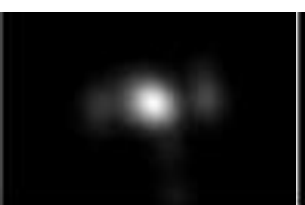

(b) Human

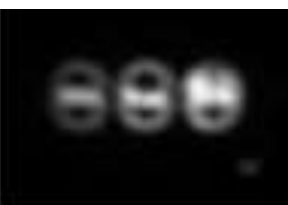

(g) PQFT

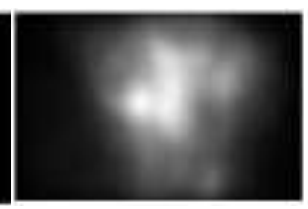

(c) HEVC

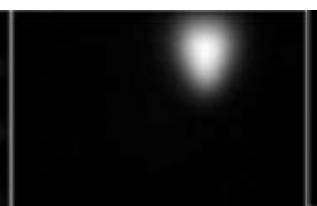

(h) Rudoy

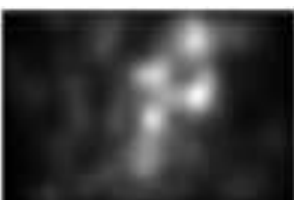

(c) HEVC

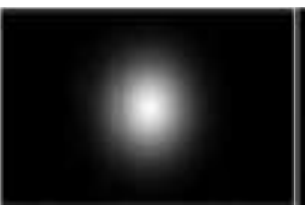

(h) Rudoy

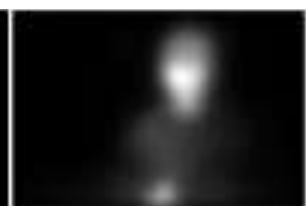

(c) HEVC

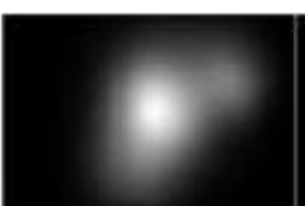

(h) Rudoy

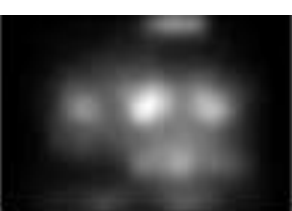

(c) HEVC

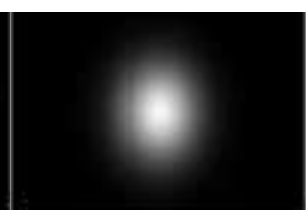

(h) Rudoy

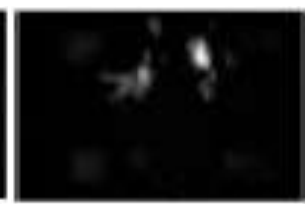

(d) itti

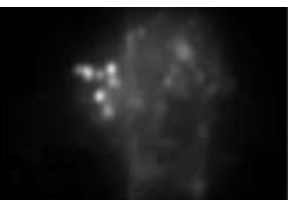

(i) Fang

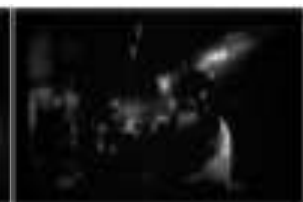

(d) itti

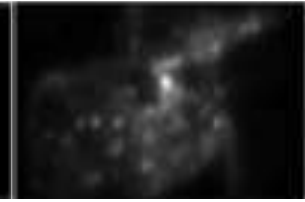

(i) Fang

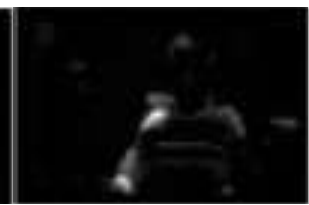

(d) itti

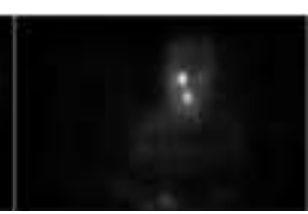

(i) Fang

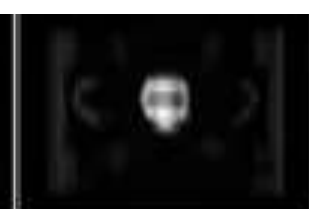

(d) itti

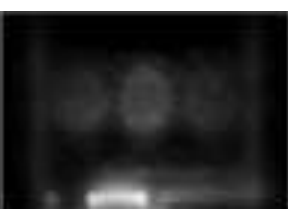

(i) Fang

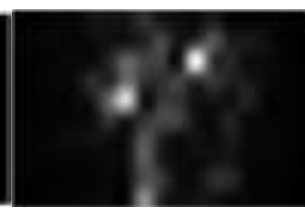

(e) Surprise

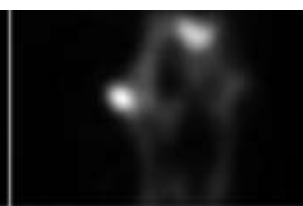

(j)OBDL

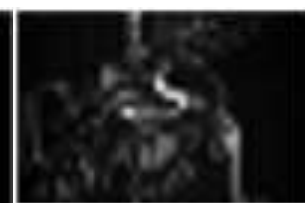

(e) Surprise

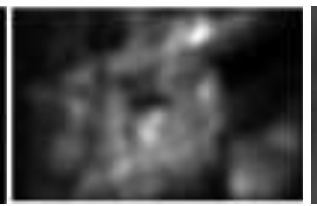

(j)OBDL

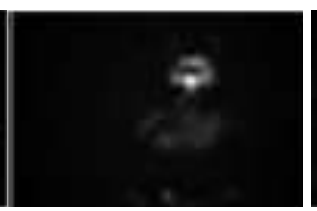

(e) Surprise

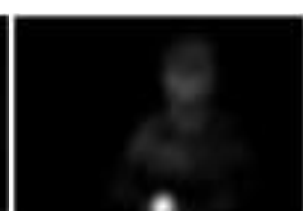

(j)OBDL

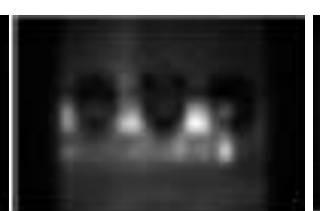

(e) Surprise

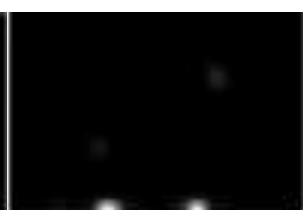

(j)OBDL

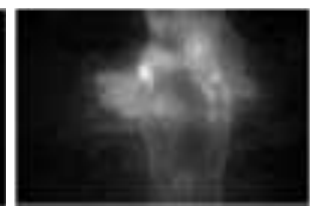

(f) Judd

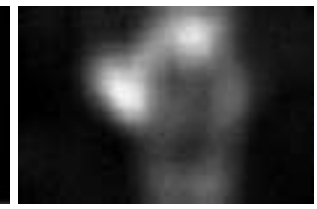

(k) OUR

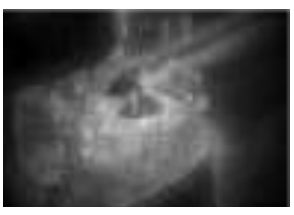

(f) Judd

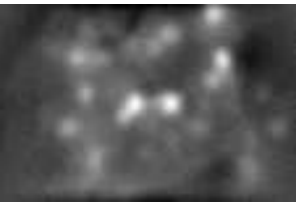

(k) OUR

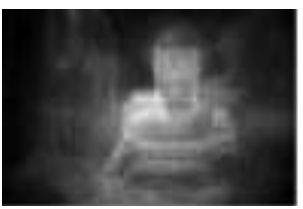

(f) Judd

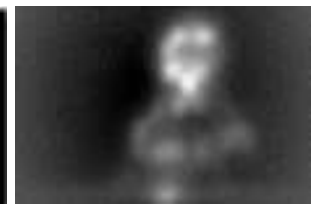

(k) OUR

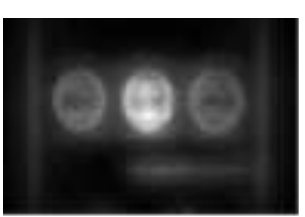

(f) Judd

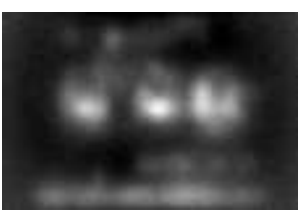

(k) OUR
The Table.3 Saliency maps of RaceHorseD video selected from the first time of our cross validation experiments. The maps were yielded by our and other 7 methods as well the ground-truth human fixations. Note that the results of only one frame are shown 
for selected video. (a) Input (b) Human (c) hevc (d) Itti (e) Surprise (f) Judd (g) PQFT (h) Rudoy (i) Fang (j) OBDL and (k) author proposed work. Our experimental results shows the better outcomes in comparison either other techniques. Therefore, it produces a better saliency map.

\section{CONCLUSION}

The 21 st century has provided an enormous evolution in the field of High Definition videos. However, there are few problems associated with it, which cannot be ignore. Therefore, to reduce these drawbacks we have implemented an efficient video compression technique as modified HEVC coding based on saliency features. In this paper, we have estimated saliency, considering HEVC video_database dataset. HEVC video_database contains total 33 videos. Here, we have shown saliency map comparison with other existing techniques for Race HorseD for videos in Table.3. Our experimental results outperforms all the existing techniques in terms of saliency map detection, AUC, NSS, KLD and JSD which shown in Table. 1 and Table.2. The average AUC, NSS and KLD value by our proposed method are $0.846,1.702$ and 0.532 respectively which is very high compare to other existing technique. Similarly, the Slideshow video gives highest JSD as 0.615 using our proposed method. These results verify that our model is more efficient than any other techniques. In future, this model can be used in the field of medical, photography, satellite imaging, HDTV, stereoscopic video processing, face recognition and video coding or encoding.

\section{REFERENCES}

[1] Cisco, "Cisco Visual Networking Index: Forecast and Methodology", Available at: https://www. cisco. com/c/en/us/solutions/collateral/service-provider/visualnetworking-index-vni/complete-white-paper-c11-481360. html.

[2] J. Ostermann et al., "Video Coding with H.264/AVC: Tools, Performance, and Complexity", IEEE Circuits and Systems Magazine, Vol. 4, No. 4, pp. 7-28, 2004.

[3] G.J. Sullivan, J.R. Ohm, W. Han and T. Wiegand, "Overview of the High Efficiency Video Coding (HEVC) Standard", IEEE Transactions on Circuits and Systems for Video Technology, Vol. 22, No. 12, pp. 1649-1668, 2012.

[4] R.C. Reininger and J.D. Gibson, "Distributions of the TwoDimensional DCT Coefficients for Images", IEEE Transactions on Communications, Vol. 31, No. 6, pp. 835839, 1983.

[5] S.R. Smooth and R.A. Rowe, "Study of DCT Coefficients Distributions", Proceedings of International Conference on Human Vision and Electronic Imaging, pp. 365-368, 1996.

[6] N. Kamaci, Y. Altunbasak and R.M. Merereau, "Frame Bit Allocation for the H.264/AVC Video Coder Via CauchyDensity-based Rate and Distortion Models", IEEE Transactions on Circuits and Systems for Video Technology, Vol. 15, No. 8, pp. 994-1006, 2005.

[7] Z. He, Y.K. Kim and S.K. Mitra, "Low-Delay Rate Control for DCT Video Coding Via $\rho$-Domain Source Modeling", IEEE Transactions on Circuits and Systems for Video Technology, Vol. 11, No. 8, pp. 928-940, 2001.
[8] J. Sun, Y. Duan, J. Li, J. Liu and Z. Guo, "Rate-Distortion Analysis of Dead-Zone Plus Uniform Threshold Scalar Quantization and its Application-Part II: Two-Pass VBR Coding for H.264/AVC", IEEE Transactions on Image Processing, Vol. 22, No. 1, pp. 215-228, 2013.

[9] J. Hou, S. Wan, Z. Ma and L.P. Chau, "Consistent Video Quality Control in Scalable Video Coding using Dependent Distortion Quantization Model", IEEE Transactions on Broadcasting, Vol. 59, No. 4, pp. 717-724, 2013.

[10] Y.H. Tan, C. Yeo and Z. Li, "Single-Pass Rate Control with Texture and Non-Texture Rate-Distortion Models", IEEE Transactions on Circuits and Systems for Video Technology, Vol. 22, No. 8, pp. 1236-1245, 2012.

[11] C.Y. Wu and P.C. Su, "A Content-Adaptive DistortionQuantization Model for H.264AVC and its Applications", IEEE Transactions on Circuits and Systems for Video Technology, Vol. 24, No. 1, pp. 113-126, 2014.

[12] M. Tagliasacchi, G. Valenzise and S. Tubaro, "Minimum Variance Optimal Rate Allocation for Multiplexed $\mathrm{H}$. 264/AVC Streams", IEEE Transactions on Image Processing, Vol. 17, No. 7, pp. 1129-1143, 2008.

[13] Myunghoon Jeon, Namgi Kim and Byoung-Dai Lee, "MapReduce-based Distributed Video Encoding using Content-Aware Video Segmentation and Scheduling", IEEE Access, Vol. 4, pp. 6802-6815, 2016.

[14] A. Ilic, S. Momcilovic, N. Roma and L. Sousa, "Adaptive Scheduling Framework for Real-Time Video Encoding on Heterogeneous Systems", IEEE Transactions on Circuits and Systems for Video Technology, Vol. 26, No. 3, pp. 597611, 2016.

[15] K.L. Chung, Y.H. Huang, C.H. Lin and J.P. Fang, "Novel Bitrate Saving and Fast Coding for Depth Videos in 3DHEVC", IEEE Transactions on Circuits and Systems for Video Technology, Vol. 26, No. 10, pp. 1859-1869, 2016.

[16] G. Kim, K. Yi and C.M. Kyung, "A Content-Aware Video Encoding Scheme Based on Single-Pass Consistent Quality Control”, IEEE Transactions on Broadcasting, Vol. 62, No. 4, pp. 800-816, 2016.

[17] J. Chao and E. Steinbach, "Keypoint Encoding for Improved Feature Extraction From Compressed Video at Low Bitrates", IEEE Transactions on Multimedia, Vol. 18, No. 1, pp. 25-39, 2016.

[18] Hadi Hadizadeh, Mario J. Enriquez and Ivan V. Bajic, "EyeTracking Database for a Set of Standard Video Sequences", IEEE Transactions on Image Processing, Vol. 21, No. 2, pp. 898-903, 2012.

[19] M. Xu, L. Jiang, X. Sun, Z. Ye and Z. Wang, "Learning to Detect Video Saliency With HEVC Features", IEEE Transactions on Image Processing, Vol. 26, No. 1, pp. 369$385,2017$.

[20] G. Sullivan, J. Ohm, W.J. Han, and T. Wiegand. "Overview of the High Efficiency Video Coding (HEVC) Standard", IEEE Transactions on Circuits and Systems for Video Technology, Vol. 22, No. 12, pp. 1649-1668, 2012.

[21] T. Wiegand, G.J. Sullivan, G. Bjontegaard and A. Luthra, "Overview of the H. 264/AVC Video Coding Standard", IEEE Transactions on Circuits and Systems for Video Technology, Vol. 13, No. 7, pp. 560-576, 2003.

[22] M.G. Arvanitidou, A. Glantz, A. Krutz, T. Sikora, M. Mrak and A. Kondoz, "Global Motion Estimation using Variable 
Block Sizes and its Application to Object Segmentation", Proceedings of $10^{\text {th }}$ Workshop on Image Analysis for Multimedia Interactive Services, pp. 173-176, 2009.

[23] L. Itti, "Automatic foveation for Video Compression using a Neurobiological Model of Visual Attention", IEEE Transactions on Image Processing, Vol. 13, No. 10, pp. 1304-1318, 2004.

[24] L. Itti, C. Koch and E. Niebur, "A Model of Saliency-based Visual Attention for Rapid Scene Analysis", IEEE Transactions on Pattern Analysis and Machine Intelligence, Vol. 20, No. 11, pp. 1254-1259, 1998.

[25] L. Itti and P. Baldi, "Bayesian Surprise Attracts Human Attention", Vision Research, Vol. 49, No. 10, pp. 12951306, 2009.

[26] T. Judd, K. Ehinger, F. Durand and A. Torralba, "Learning to Predict where Humans Look", Proceedings of $10^{\text {th }}$ International Conference on Computer Vision, pp. 21062113, 2009.

[27] C. Guo and L. Zhang, "A Novel Multiresolution Spatiotemporal Saliency Detection Model and its Applications in Image and Video Compression", IEEE Transactions on Image Processing, Vol. 19, No. 1, pp. 185198, 2010.

[28] D. Rudoy, D.B. Goldman, E. Shechtman, and L. ZelnikManor, "Learning Video Saliency from Human Gaze using Candidate Selection", Proceedings of IEEE Conference on Computer Vision and Pattern Recognition, pp. 1147-1154, 2013.

[29] Y. Fang, W. Lin, Z. Chen, C.M. Tsai and C.W. Lin, "A Video Saliency Detection Model in Compressed Domain”,
IEEE Transactions on Circuits and Systems for Video Technology, Vol. 24, No. 1, pp. 27-38, 2014.

[30] S. Hossein Khatoonabadi, N. Vasconcelos, I.V. Bajic and Y. Shan, "How Many Bits does it take for a Stimulus to be Salient?", Proceedings of IEEE Conference on Computer Vision and Pattern Recognition, pp. 5501-5510, 2015.

[31] Mai Xu, Xin Deng, Shengxi Li and Zulin Wang, "Regionof-Interest based Conversational HEVC Coding with Hierarchical Perception Model of Face", IEEE Journal of Selected Topics in Signal Processing, Vol. 8, No. 3, pp. 475489, 2014.

[32] J.R. Ohm, G.J. Sullivan, H. Schwarz, T.K. Tan and T. Wiegand, "Comparison of the Coding Efficiency of Video Coding Standards-Including High Efficiency Video Coding (HEVC)", IEEE Transactions on Circuits and Systems for Video Technology, Vol. 22, No. 12, pp. 1669-1684, 2012.

[33] Ali Borji and Laurent Itti, "State-of-the-Art in Visual Attention Modeling", IEEE Transactions on Pattern Analysis and Machine Intelligence, Vol. 35, No. 1, pp. 185207, 2013.

[34] Ali Borji, Dicky N. Sihite and Laurent Itti, "Quantitative Analysis of Human-Model Agreement in Visual Saliency Modeling: A Comparative Study", IEEE Transactions on Image Processing, Vol. 22, No. 1, pp. 55-69, 2013.

[35] L. Itti and P. Baldi, "A Principled Approach to Detecting Surprising Events in Video", Proceedings of IEEE Computer Society Conference on Computer Vision and Pattern Recognition, Vol. 1, pp. 631-637, 2005. 